\title{
Nonlinear-optical probing of nanosecond ferroelectric switching
}

\author{
E. D. Mishina, N. E. Sherstyuk, V. I. Stadnichuk, and A. S. Sigov \\ Moscow Institute of Radioengineering, Electronics and Automation, Moscow 117454, Russia
}

V. M. Mukhorotov and Yu. I. Golovko

Institute of General Physics, Russian Academy of Science, Moscow, Russia

\author{
A. van Etteger and Th. Rasing ${ }^{\text {a) }}$ \\ University of Nijmegen, NSRIM Institute, Nijmegen, The Netherlands
}

(Received 21 April 2003; accepted 23 July 2003)

It is demonstrated that the switching dynamics of the ferroelectric polarization in thin $\mathrm{Ba}(\mathrm{SrTi}) \mathrm{O}_{3}$ (BST) films can be followed by optical second harmonic generation with a time resolution that is only limited by the gating electronics. It is shown that the characteristic time of electric-field induced polarization switching in $70 \mathrm{~nm}$ BST films is less than $\tau=5 \mathrm{~ns}$. (C) 2003 American Institute of Physics. [DOI: 10.1063/1.1612905]

Switching of thin ferroelectric films is intensively studied because of their main applications, random access memories, and electro-optical modulators. ${ }^{1,2}$ The switching requirements for memory and modulator applications are different: in a memory one needs to switch a stable remanent polarization across a coercive field (hysteresis regime) whereas for a modulator one needs a completely reversible polarization switching (quasilinear regime). Nevertheless, the next generation of both types of devices should operate at frequencies of 1-100 GHz. Since the switching speed is determined by the dynamics of ferroelectric domains under applied electric field, ${ }^{3}$ the dynamical response of the domains should be studied on a time scale from nanoseconds down to picoseconds.

Usually, information on domain relaxation kinetics in a ferroelectric film is derived from dielectric measurements. The shortest time measured in this way is $1.8 \mathrm{~ns} .{ }^{4}$ However, dielectric measurements can be affected by transient characteristics of electric circuits. ${ }^{3}$ Circuit-independent information can be obtained by optical and x-ray techniques. ${ }^{5}$ Both electro-optical and nonlinear optical techniques may give direct information on the domain switching. However, the electric-field induced birefringence is superimposed on the natural birefringence and is very small $\left(\Delta n \sim 10^{-4}\right)$. Of course a birefringence modulation influences the nonlinear optical response as well. But this influence is minor in comparison with the change in nonlinear-optical susceptibility, which may increase up to several orders of magnitude while the domain configuration changes from average centrosymmetric (antiparallel compensated domains) to noncentrosymmetric (parallel domains). In this way optical second harmonic generation (SHG) gives direct information about domain orientation and switching. ${ }^{6,7}$ However, applications of SHG to study domain orientation were limited to quasisteady state (low frequency) studies.

In this letter we report experimental studies of fast polarization switching in a ferroelectric thin $\mathrm{Ba}(\mathrm{SrTi}) \mathrm{O}_{3}$ (BST)

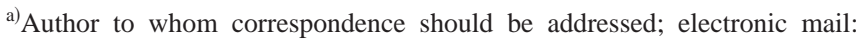
theoras@sci.kun.nl
}

film by optical second harmonic generation with a time resolution of about $1 \mathrm{~ns}$. In the frame of a simple model the time constant of the polarization switching is estimated and deviations from the model calculations are discussed.

The BST films of $70 \mathrm{~nm}$ thickness were deposited on $\mathrm{MgO}(100)$ substrates by rf sputtering from a ceramic target (see for details Ref. 7). X-ray diffraction revealed only (001) type reflections (corresponding to $c$-oriented domains), no evidence of second-phase nucleation was found. The barium concentration in the films is 0.7 , which gives a Curie temperature $T_{C}=15{ }^{\circ} \mathrm{C}$ for bulk BST. For a thin film of about several tens of nanometer the phase transition becomes dispersed ${ }^{8}$ which allows measuring ferroelectric properties at room temperature.

For electrical measurements two copper semidisks were evaporated on top of the film with a gap of $25 \mu \mathrm{m}$ between them, providing an in-plane configuration of the external field [see Figs. 1(a) and 1(b)]. For fast switching the output of a pulse generator was used with a pulse rise time of $\sim 5 \mathrm{~ns}$, pulse width of $\sim 20 \mathrm{~ns}$, and maximum voltage of $\pm 15 \mathrm{~V}$, providing an electric field strength $E_{L}=6$ $\times 10^{5} \mathrm{~V} / \mathrm{m}$. Two types of voltage pulses were used (see Fig. 2 , lines). The first one, denoted hereafter as a "normal" pulse with voltage increasing from zero to maximum amplitude [positive, Fig. 2(a) or negative, Fig. 2(b)] followed by a decrease to zero. The second one, "zero" pulse with the voltage drop to zero from a constant value [positive, Fig. 2(c) or negative, Fig. 2(d)] followed by a complete voltage recover.

For conventional polarization measurements a sine-wave was applied to the sample. Corresponding hysteresis loops measured using a Sawyer-Tower circuit at $1 \mathrm{kHz}$ for two amplitudes of the external field $\left(E_{S}=2 \times 10^{7} \mathrm{~V} / \mathrm{m}\right.$, saturating regime and $E_{L}=6 \times 10^{5} \mathrm{~V} / \mathrm{m}$, quasilinear regime) are shown in Fig. 1(c).

For the SHG measurements the output of a Ti:sapphire laser at $760 \mathrm{~nm}$ with a pulse width of about $100 \mathrm{fs}$ and a repetition rate of $82 \mathrm{MHz}$ was used, with an average power of $100 \mathrm{~mW}$ focused onto a spot of about $10 \mu \mathrm{m}$. Details of the SHG setup can be found in Ref. 7. A transmission geometry was used and the angle of incidence was varied by rotating the sample around the vertical in-plane axis in the 

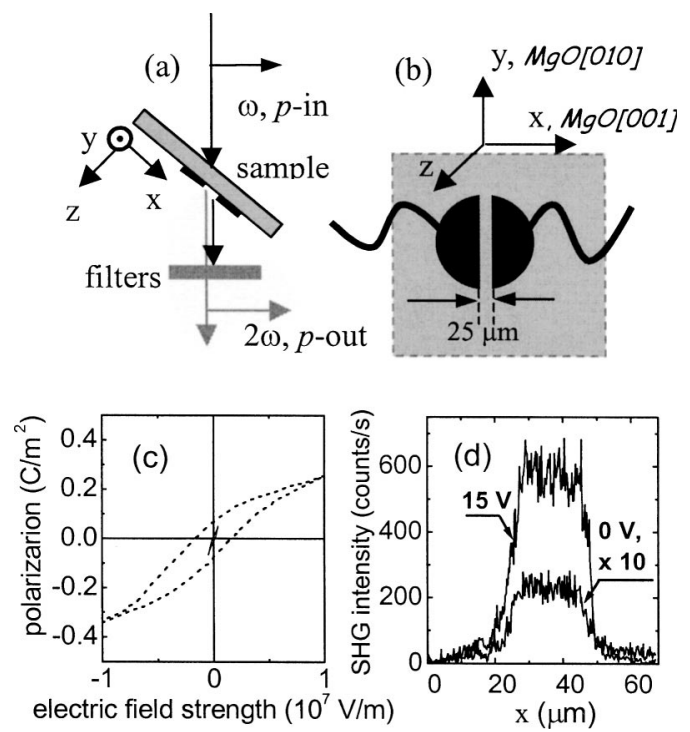

FIG. 1. (a) Optical experimental geometry: $x y z$ is the sample frame, the fundamental and SHG waves propagate at $45^{\circ}$ with respect to the $z$ axis, and fundamental wave is blocked by filters; (b) electrode configuration; (c) typical hysteresis loops obtained through Sawyer-Tower measurements in saturation $\left(E_{S}=2 \times 10^{7} \mathrm{~V} / \mathrm{m}\right.$, dashed line $)$ and quasilinear $\left(E_{L}=6 \times 10^{5} \mathrm{~V} / \mathrm{m}\right.$, solid line) regimes, $f=1 \mathrm{kHz}$; and (d) the SHG scans along the normal to the gap between the electrodes for unbiased and biased film.

range of $\pm 35^{\circ}$. In order to check that the detected SHG signal came only from the ferroelectric film and not the metal electrodes the sample was scanned across the gap. Figure 1(d) shows that the SHG signal from the electrodes is more than two orders of magnitude smaller than that from the film. In order to measure ferroelectric switching with the SHG probe, a gated photon-counting system was used with a gate width $\tau_{\text {gate }}=5 \mathrm{~ns}$. The gate was triggered by the pulse generator with a repetition rate $f=100 \mathrm{kHz}$, and the gate delay was varied within $\tau_{\text {ext }}=1 / f$ with a step $\tau_{\text {del }}$ up to 300 ns. Averaging time for data acquisition for each delay was 10 s.

Figure 2 shows the SHG transients during the application of an external voltage pulse in the linear regime. For the normal pulse the SHG signal follows quite close the voltage transient and the SHG oscillations decay simultaneously with the voltage decay (note that the optical signal is proportional to the polarization squared, see later). For the zero pulses the
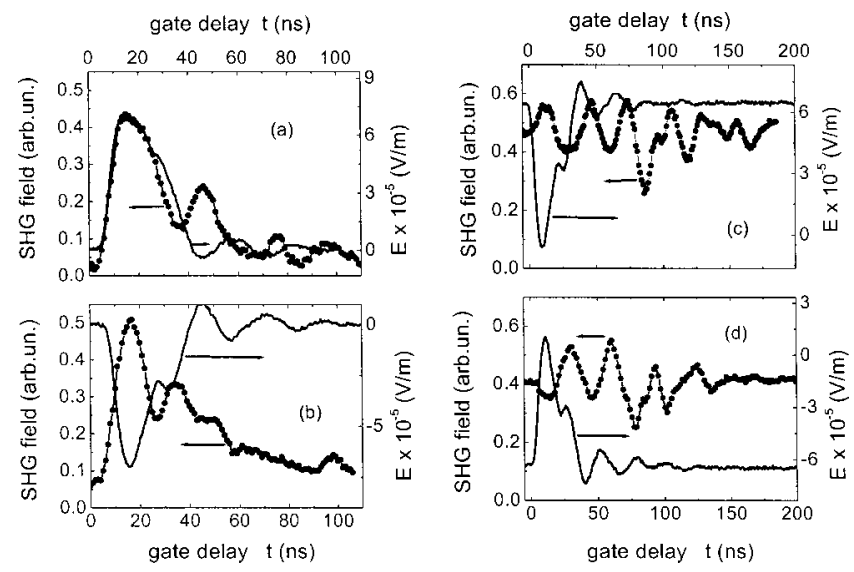

FIG. 2. Electric field (lines) and SHG transients (points) for normal positive (a), negative (b), zero positive (c), and negative (d) pulses. Delay time step is $\Delta \tau_{\mathrm{del}}=2 \mathrm{~ns}$.

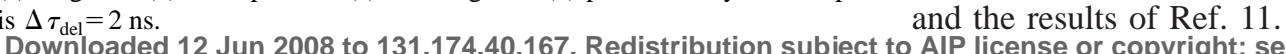

SHG signal reveals strong oscillations even after the voltage has completely decayed. The modulation depth of the SHG signal reaches $75 \%$ [Fig. $2(\mathrm{c}), \tau_{\text {del }}=90 \mathrm{~ns}$ ], while the modulation depth of the voltage is not larger than $5 \%$ for the same delay. For both normal and zero pulses no difference was observed in the SHG transients with a change of the angle of incidence in the range of $\pm 35^{\circ}$.

Since crystallographically the films are $c$ oriented with a high degree of accuracy, without applied field the SHG signal is determined by contributions of $c$ domains. Applying an in-plane field causes a switching to the in-plane $a$-domain configuration. Figure 2 shows that for $E=0(t=0)$ the SHG field is about an order of magnitude smaller than for the maximum value of $E(t=15 \mathrm{~ns})$. This means that for a biased film the contribution from $c$ domains can be neglected. Simultaneous damping of voltage and SHG oscillations means that no remanent polarization appears after the normal pulse, thus the switching in this case indeed occurs in the quasilinear regime.

In the frame of the Landau theory, the evolution of the ferroelectric polarization in the bulk of a film can be described by ${ }^{9}$

$$
\tau_{D} \frac{d P_{x}}{d t}=A P_{x}+B P_{x}^{3}+\varepsilon_{0} E_{x},
$$

where $P_{x}$ is the in-plane component of the dielectric polarization, $A$ and $B$ are the coefficients of the thermodynamic potential expansion in powers of $P, E_{x}$ is the electric field strength, $T_{C}$ is the Curie temperature, $\varepsilon_{0}$ is the permittivity of free space, and $\tau_{D}$ is the Debye relaxation time. If the local polarization within a single domain $p_{x}^{+}$does not depend on the electric field (an assumption which is valid for electric field strengths far away from the saturation value), then the ferroelectric polarization equals the volume domain fraction: $P_{x}=\int\left(p_{x}^{+}-p_{x}^{-}\right) d V=\Delta F_{v x}$. The square root of the coherent SHG intensity is proportional to the in-plane volume domain fraction (and thus to the absolute value of ferroelectric polarization $)^{6,7}$

$$
\sqrt{I(2 \omega)} \propto\left|\Delta F_{v x}\right|=\left|P_{x}\right| .
$$

Equations (1) and (2) give the temporal dependence of the SHG intensity. Results of the model calculations are presented in Fig. 3. The voltage pulse (shown in Fig. 3 by thick lines) was reconstructed by superposition of Gaussian and $\sin x / x$ functions. The coefficients $A$ and $B$ in Eq. (1) used for the simulations were taken from Ref. 10 for $\mathrm{BaTiO}_{3}(A$ $\left.=10^{-5}, B=-0.5 \mathrm{~m}^{2} / \mathrm{C}^{4}\right)$. Simulated hysteresis loops in both saturating and quasilinear regimes $\left(E_{S}=2 \times 10^{7} \mathrm{~V} / \mathrm{m}\right.$, and $E_{L}=6 \times 10^{5} \mathrm{~V} / \mathrm{m}$, respectively) are presented in Fig. 3(a). Qualitatively the calculated loops are similar to the experimental ones. The SHG transients for different values of $\tau_{D}$ are presented in Fig. 3(b). The best simulation for the normal-pulse switching experiments were obtained for $\tau_{D}$ $=0.1 \mathrm{ps}$. Switching time in the vicinity of $T_{C}$ can be calculated as ${ }^{11} \tau_{S} \propto \varepsilon \tau_{D}$, where $\varepsilon$ is the dielectric permittivity of the film. For $\mathrm{BST}^{12} \varepsilon \sim 500-5000$, which gives $\tau_{S}$ $=0.05-0.5 \mathrm{~ns}$, which is consistent with our measurements ( $\tau_{S}$ is smaller than the temporal resolution of the experiment)

AIP license or copyright; see http://apl.aip.org/apl/copyright.jsp 

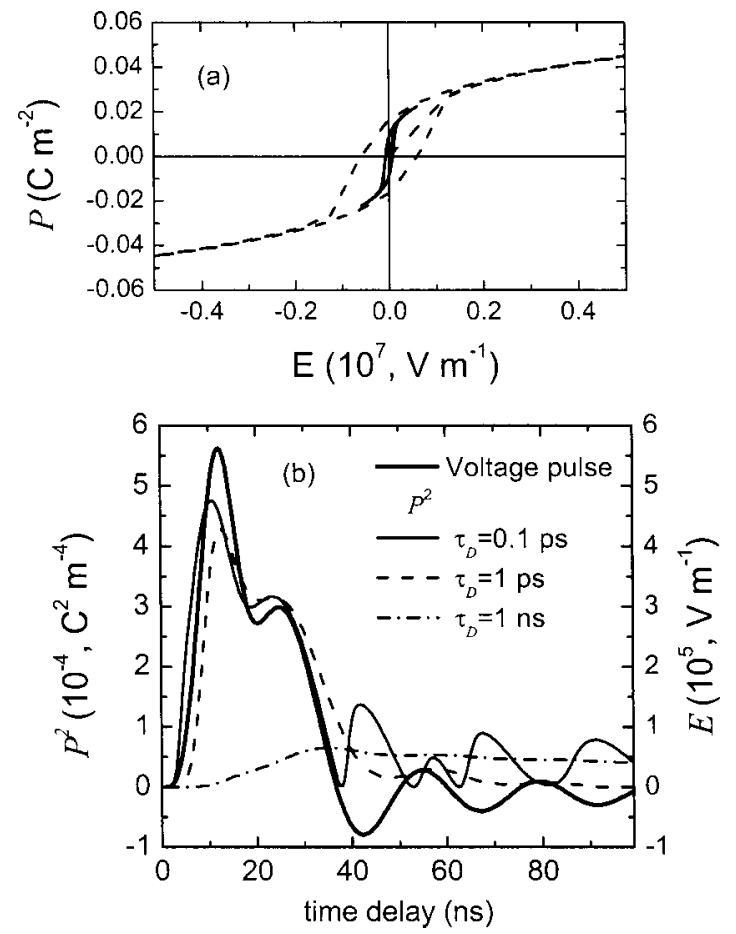

FIG. 3. (a) Simulated electric field (thick line) and SHG (thin lines) transients corresponding to the normal voltage pulse and (b) simulated dielectric hysteresis for normalized polarization for saturating (dashed) and quasilinear (solid line) regime. For all simulations (see Ref. 10) $A=10^{-5}, B=$ $-0.5 \mathrm{~m}^{2} / \mathrm{C}^{4}$.

Calculations show that the phase shift of the oscillations directly follows from the model; additional maxima appear in SHG intensity because it depends on the second power of the polarization. Simulations for the zero pulse give the same shape of the SHG transients, and no conditions were found yielding an increase of the SHG oscillations during the decrease of the voltage oscillations. This means that the results for the zero pulse switching cannot be understood in this way.

These results beautifully demonstrate how the nonlinear optical technique of SHG can be used to probe the time evolution of the polarization dynamics under the influence of an electric field pulse, with a time resolution that in principal is limited only by the laser pulse width (in the present experiment, the limiting factor was the electronic response of the detection system).

As far as the surprising results for the zero pulse are concerned, these might be due to the generation of an oscillating piezoresponse. In perovskite complex oxides a variety of nonlinear surface acoustic effects have been observed due to their piezoelectric properties. These waves may propagate as free acoustic waves or solitons ${ }^{13}$ and such stress modula- tion may influence the nonlinear optical susceptibility during the acoustic wave propagation. ${ }^{14}$ Fourier transform of the SHG signal gives a main frequency of the SHG oscillations of $33 \mathrm{MHz}$, which coincides with the inverse of the electric pulse width. The strong response that is observed after the electric field pulse decayed might be due to an accidental acoustic resonance with this frequency.

In conclusion, we have shown the capability of SHG to measure the switching process in ferroelectric thin films. In a $70 \mathrm{~nm}$ BST film the switching time is shown to be not larger than the rise time of the external voltage pulse (5 ns). Two types of switching/relaxation processes were observed. When a normal voltage pulse is applied to the film, the SHG transient follows the voltage transient and the temporal behavior of the ferroelectric polarization can be explained in the frame of a simple Landau model. This regime can be used for fast optical modulator applications. For a zero pulse, oscillations of the SHG signal are observed that extend far beyond the applied voltage pulse and that cannot be explained in the frame of existing theories. The generation of acoustic waves may be responsible for this observation, but a detailed explanation is still lacking.

This work is supported by the Dutch Science Foundation NWO (Grant No. 1604-1999), Russian Foundation of Basic Research (Grant No. 03-02-16945), INTAS (Grant No. 010075). The authors would like to thank R. Gelsing for sample preparation.

${ }^{1}$ S. Li, H. Zheng, L. Salamanca-Riba, R. Ramesh, I. Naumov, and K. Rabe, Appl. Phys. Lett. 81, 4398 (2002).

${ }^{2}$ J. Im, O. Auciello, P. K. Baumann, S. K. Streiffer, D. Y. Kaufman, and A. R. Krauss, Appl. Phys. Lett. 76, 625 (2000).

${ }^{3}$ J. F. Scott, Ferroelectr. Rev. 1, 1 (1998).

${ }^{4}$ P. K. Larsen, G. L. M. Kampschöer, M. J. E. Ulenaers, G. A. C. M. Spierings, and R. Cuppens, Appl. Phys. Lett. 59, 611 (1991).

${ }^{5}$ E. Zolotoyabko, J. P. Quintana, B. H. Hoermann, and B. W. Wessels, Appl. Phys. Lett. 80, 3159 (2002).

${ }^{6}$ V. Gopalan and R. Raj, Appl. Phys. Lett. 68, 1323 (1996).

${ }^{7}$ E. D. Mishina, N. E. Sherstyuk, D. R. Barskiy, A. S. Sigov, Yu. I. Golovko, V. M. Mukhorotov, M. De Santo, and Th. Rasing, J. Appl. Phys. 93, 6216 (2003).

${ }^{8}$ V. Mukhortov, Y. Golovko, V. Sviridov, V. Mukhortov, V. Alyoshin, and V. Dudkevich, Phys. Status Solidi A 77, K37 (1983).

${ }^{9}$ R. Blinc and B. Z. Ks , Soft Modes in Ferroelectrics and Antiferroelectrics (North-Holland, Amsterdam, 1974).

${ }^{10} \mathrm{~S}$. Li, J. A. Eastman, J. M. Vetrone, C. M. Foster, R. E. Newnham, and L. E. Cross, Jpn. J. Appl. Phys., Part 1 36, 5169 (1997).

${ }^{11}$ V. V. Kolesnikov, A. T. Kozakov, and A. V. Nikol'skii, Phys. Solid State 42, 146 (2000).

${ }^{12}$ W. Chang, C. M. Gilmore, W.-J. Kim, J. M. Pond, S. W. Kirchoefer, S. B. Qadri, and D. B. Chirsey, J. Appl. Phys. 87, 3044 (2000).

${ }^{13}$ V. Kavalerov, T. Fujii, and M. Inoue, J. Appl. Phys. 87, 907 (2000).

${ }^{14}$ T. Zhao, H. Lu, F. Chen, G. Yang, and Z. Chen, J. Appl. Phys. 87, 7448 (2000) 